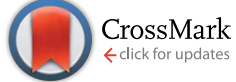

Cite this: RSC Adv., 2015, 5, 34517

\title{
Quick synthesis, functionalization and properties of uniform, luminescent $\mathrm{LuPO}_{4}$-based nanoparticles $\uparrow$
}

\author{
Ana I. Becerro* and Manuel Ocaña
}

The aim of this study was to find a surfactant-free method for the synthesis of uniform Eu:LuPO 4 nanophosphors which are able to form stable colloidal suspensions in aqueous media. Uniform, ovoid Eu-doped $\mathrm{LuPO}_{4}$ fluorescent nanoparticles were obtained after aging for 30 minutes at $180{ }^{\circ} \mathrm{C}$ a butylene glycol solution containing, exclusively, lutetium acetate, europium acetate and $\mathrm{H}_{3} \mathrm{PO}_{4}$. $\mathrm{XRD}$ and digital diffraction patterns of HRTEM images suggested that the particles were single crystalline in nature with the $c$-axis of the unit cell parallel to the long particle axis. The luminescence study revealed that the optimum doping level was 5 molar\%. The latter particles (85 nm $\times 40 \mathrm{~nm}$ dimensions) were functionalized with polyacrylic acid and their colloidal stability in two different biological buffers was demonstrated to persist for at least 15 days.

Received 25th March 2015 Accepted 8th April 2015

DOI: $10.1039 / \mathrm{c} 5 \mathrm{ra05305f}$

www.rsc.org/advances
$300 \mathrm{~nm}$, well above the size of nanometer-scale materials. To the best of our knowledge, only two papers have reported the synthesis of monodisperse, nanometer-size $\mathrm{LuPO}_{4}$ particles. ${ }^{15,16}$ In both cases a rather tedious synthesis method was used based on the use of several organic additives. The inconvenience of these additives is that they remain attached to the surface of the particles making them hydrophobic. A challenge for bioanalytical or therapeutic applications of this material is to find new synthesis routes to produce nanoparticles that can be dispersed in aqueous media to form stable colloidal solutions.

In the present study, we report a quick and really simple method to synthesize uniform Eu-doped $\mathrm{LuPO}_{4}$ nanometer size particles, with optimized luminescent properties. The method is based on a homogeneous precipitation process in a solution containing, exclusively, $\mathrm{Ln}^{3+}\left(\mathrm{Lu}^{3+}, \mathrm{Eu}^{3+}\right)$ acetates and $\mathrm{H}_{3} \mathrm{PO}_{4}$. We have investigated the influence of the precursor nature and concentration as well as the type of solvent on the morphology of the precipitated particles. The particles have eventually been functionalized with polyacrylic acid (PAA) to demonstrate that they can be easily surface-modified to provide anchor sites for functional ligands. ${ }^{17}$ Finally, the colloidal stability of the PAAfunctionalized particles has been analyzed in different biological buffers (MES and PBS).

\section{Experimental section}

\subsection{Synthesis of samples}

The $\mathrm{LuPO}_{4}$ nanoparticles were obtained according to the following method: lutetium acetate hydrate $\left(\mathrm{Lu}\left(\mathrm{CH}_{3} \mathrm{COO}\right)_{3} \cdot x \mathrm{H}_{2} \mathrm{O}\right.$, Sigma Aldrich, 99.9\%) was dissolved, with magnetic stirring, in $10 \mathrm{~mL}$ of butylene glycol (BG, Fluka, 99.5\%) to obtain a $0.025 \mathrm{M}$ solution. The solution was admixed with $\mathrm{H}_{3} \mathrm{PO}_{4}(0.1 \mathrm{M})$ and magnetically stirred for 5 minutes at room temperature to favor 
homogenization. The final solution was aged for 30 minutes in tightly closed test tubes using an oven preheated at $180{ }^{\circ} \mathrm{C}$. The resulting dispersion was cooled down to room temperature, centrifuged to remove the supernatants and washed, twice with ethanol and once with double distilled water. For some analyses, the powder was dried at room temperature. Other values of aging time (from 4 to 30 minutes) as well as different $\mathrm{Lu}$ and $\mathrm{PO}_{4}$ sources were used to analyze their effect on the particles characteristics. Such sources were $\mathrm{Lu}\left(\mathrm{NO}_{3}\right)_{3}$ (Sigma Aldrich, 99.99\%) and 1-butyl-3-methylimidazolium phosphate ([BMIM] $\left[\mathrm{PO}_{4}\right]$ 99\% Strem Chemicals), for $\mathrm{Lu}$ and $\mathrm{PO}_{4}$, respectively. We also used solvents other than BG to analyze their effect on the particles morphology. The solvents used were ethylene glycol (EG, Sigma Aldrich, 99.99\%) and glycerol (Gly, Sigma Aldrich > 99.5\%).

$\mathrm{The}_{\mathrm{Eu}}^{3+}$-doped particles were synthesized following the same procedure and using $\mathrm{Eu}^{3+}$ acetate hydrate $\left(\mathrm{Eu}\left(\mathrm{CH}_{3} \mathrm{COO}\right)_{3} \cdot x \mathrm{H}_{2} \mathrm{O}\right.$, Sigma Aldrich, 99.9\%) in variable amounts. The lanthanide ions $(\mathrm{Lu}+\mathrm{Eu})$ concentration was kept constant $\left(0.025 \mathrm{~mol} \mathrm{dm}^{-3}\right)$ in all experiments, whereas the $\mathrm{Eu} /(\mathrm{Lu}+\mathrm{Eu})$ molar ratio was varied from $0.5 \%$ to $15 \%$ in order to investigate the effect of this parameter on the morphological and luminescent properties of the precipitated particles.

\subsection{Functionalization of the particles with polyacrylic acid} (PAA)

The procedure for the functionalization of the $\mathrm{Eu}^{3+}$-doped $\mathrm{LuPO}_{4}$ nanoparticles was as follows: PAA (average $M_{\mathrm{w}} \sim 1800$, Aldrich) was added to solutions with adjusted $\mathrm{pH}$ (10) containing $1 \mathrm{mg}$ $\mathrm{mL}^{-1}$ of the $\mathrm{Eu}^{3+}: \mathrm{LuPO}_{4}$ nanoparticles $\left(\mathrm{PAA} / \mathrm{Eu}^{3+}: \mathrm{LuPO}_{4}\right.$ weigh ratio $=2$ ). The obtained dispersion was magnetically stirred for 1 hour at room temperature. The so functionalized nanoparticles were washed several times with milliQ water by centrifugation and finally dispersed in milliQ water.

\subsection{Characterization techniques}

The shape and size of the particles was examined by both transmission electron microscopy (TEM, Philips 200CM) and scanning electron microscopy (SEM-FEG Hitachi S4800). Particle size distributions were obtained from the micrographs by counting several hundreds of particles, using the free software ImageJ. Additional information on the size and colloidal stability of the particles in aqueous suspension $\left(0.5 \mathrm{mg} \mathrm{mL}^{-1}\right.$ of solid) was obtained from Dynamic Light Scattering (DLS) measurements. The experiments were carried out using a Malvern Zetasizer Nano-ZS90 equipment, which was used as well to measure the zeta potential of the suspensions.

The crystalline structure of the prepared particles was assessed by X-ray diffraction (XRD) using a Panalytical, X' Pert Pro diffractometer $(\mathrm{CuK} \alpha)$ with an X-Celerator detector over an angular range of $10^{\circ}<2 \theta<120^{\circ}, 2 \theta$ step width of $0.02^{\circ}$, and $10 \mathrm{~s}$ counting time. The crystallite size was calculated using the Scherrer formula from the full width at half maximum of several single reflections. To gain additional information about the crystalline features of the synthesized nanoparticles, they were also characterized by high-resolution transmission electron microscopy (HRTEM) using a FEGTEM Tecnai 20 instrument.
The TEM images were acquired with an Ultrascan X100 camera from Gatan. Digital diffraction patterns were also calculated by Fourier Transform of such HRTEM images using the Gatan Digital Micrograph software.

The incorporation of $\mathrm{Eu}^{3+}$ into the $\mathrm{LuPO}_{4}$ structure was proved by calculation of unit cell parameters of the undoped and the $\mathrm{Eu}^{3+}$-doped particles. For this purpose, the corresponding XRD patterns were analyzed using the Rietveld method with the TOPAS software (TOPAS version 4.2, Bruker AXS, 2009). Starting crystallographic parameters were taken from those reported for tetragonal $\mathrm{LuPO}_{4} \cdot{ }^{18}$ Nominal $\mathrm{Eu}^{3+}$ contents were added to the structure. Refined parameters were: scale factor, zero error, background coefficients, unit cell parameters and atomic coordinates and atomic displacement factors of the lanthanide atoms.

The infrared spectra of the powders, diluted in KBr pellets, were recorded in a Jasco FT-IR-6200 Fourier transform spectrometer.

Thermogravimetric analyses were performed in air at a heating rate of $10{ }^{\circ} \mathrm{C} \mathrm{min}^{-1}$ using a Q600 TA instrument.

The excitation and emission spectra of the $\mathrm{Eu}^{3+}$-doped $\mathrm{LuPO}_{4}$ particles, dispersed in water $\left(2.5 \mathrm{mg} \mathrm{mL}{ }^{-1}\right)$, were measured in a Horiba Jobin Yvon spectrofluorimeter (Fluorolog3). The emission spectra were transformed to the CIE color coordinates system using a $2^{\circ}$ observer.

The colloidal stability of Eu:LuPO ${ }_{4}$ nanoparticles $(0.5 \mathrm{mg}$ $\mathrm{mL}^{-1}$ of solid) suspended in different media (milliQ water, $50 \mathrm{mM}$ MES solutions (2-( $N$-morpholino)ethanesulfonic acid, Sigma, 99\%) at pH 6.5, and in $50 \mathrm{mM}$ PBS (phosphate buffered saline) solution at $\mathrm{pH}$ 7.2) was monitored by analyzing the evolution of the hydrodynamic diameter, obtained from DLS measurements, with aging time.

\section{Results and discussion}

\subsection{Morphology and crystal structure}

The optimum experimental conditions for the synthesis of luminescent $\mathrm{LuPO}_{4}$-based particles were established on the undoped material. It was found that the aging at $180{ }^{\circ} \mathrm{C}$ for 30 minutes of a butylene glycol (BG) solution containing lutetium acetate $(0.025 \mathrm{M})$ and $\mathrm{H}_{3} \mathrm{PO}_{4}(0.1 \mathrm{M})$ led to uniform ovoidlike nanoparticles (Fig. 1, top). The particle size (Fig. 1, bottom), obtained from different TEM micrographs by counting about a hundred of particles, was 130 (s.d. 19) nm for the long axis length and 62 (s.d. 9) nm for the short axis length. Although the SEM and TEM micrographs shown in Fig. 1 suggested that the particles were uniform and non-aggregated, this aspect was further confirmed by dynamic light scattering (DLS) measurements conducted on an aqueous suspension of the particles. The zeta potential measured at the original $\mathrm{pH}(\mathrm{pH}=4)$ was very close to the isoelectric point. For this reason, we increased the $\mathrm{pH}$ of the suspension to 10.3 , obtaining a zeta potential value of $-44(10) \mathrm{mV}$ and a hydrodynamic diameter of $140 \mathrm{~nm}$. These data confirm the absence of particles aggregation.

Fig. 2 shows the XRD pattern of the product shown in Fig. 1. All the reflections could be readily indexed to the tetragonal $\mathrm{LuPO}_{4}$ phase in $\mathrm{I4}_{1} /$ amd space group (ICDD 00-043-0003, PDF4+, 

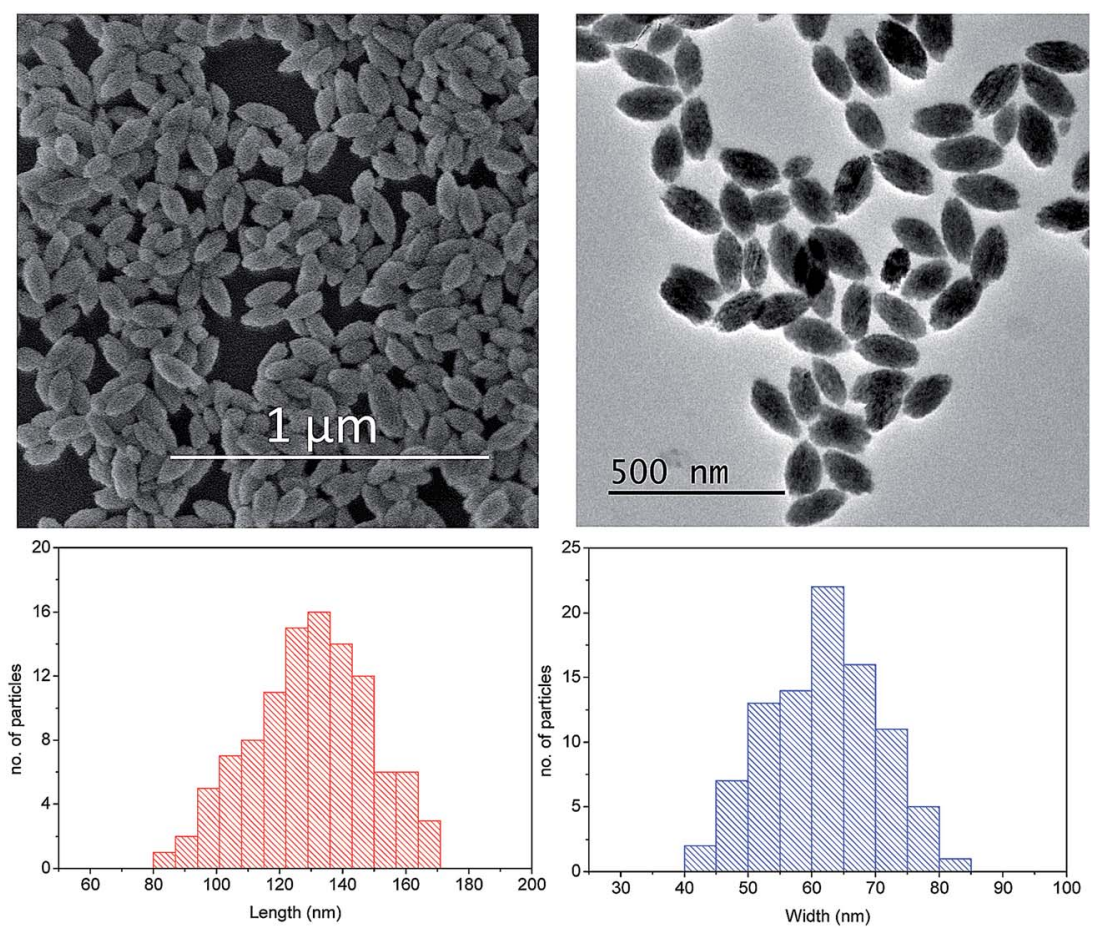

Fig. 1 SEM and TEM images of $\mathrm{LuPO}_{4}$ nanoparticles synthesized from lutetium acetate $(0.025 \mathrm{M})$ and $\mathrm{H}_{3} \mathrm{PO}_{4}(0.1 \mathrm{M})$ in $\mathrm{BG}$ solution at $180{ }^{\circ} \mathrm{C}$ for 30 minutes. Bottom: particle size distribution - length (left plot) and width (right plot) - obtained from different TEM micrographs by counting about a hundred of particles.

2013). The SEM and TEM images shown in Fig. 1 allow observing only two dimensions of the particles: their length and their width. If the particles were flat, their third dimension would be very small and they would lay with their flat faces parallel to the sample holder. This fact would notably increase the intensity of certain reflections in the XRD pattern of the sample due to the preferred orientation effect, as observed for flat particles by other authors. ${ }^{19}$ Given that all the reflections in the XRD pattern of Fig. 2 match those of the standard PDF $\left(\mathrm{LuPO}_{4}, 00-043-0003\right)$, it can be concluded that the sample is free from preferred orientation effects and that the $\mathrm{LuPO}_{4}$ particles show very likely an ovoid morphology.

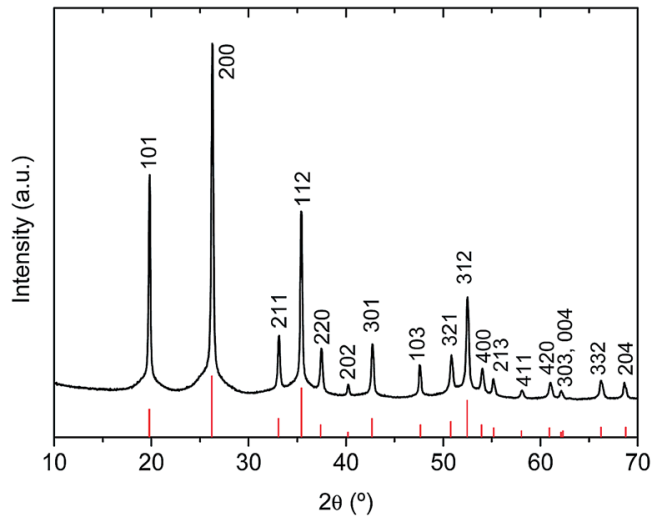

Fig. 2 XRD pattern of the particles shown in Fig. 1. The PDF 00-0430003 (tetragonal $\mathrm{LuPO}_{4}$ ) is shown on the bottom.
The crystalline domain sizes of the particles, calculated using the Scherrer formula on the (200) reflection were of $\sim 70$ $\mathrm{nm}$ and $\sim 120 \mathrm{~nm}$ on the (101) reflection. These values are very close to the particles dimensions inferred from the TEM micrographs and suggest that the particles could be single crystals in nature. In fact, a close inspection of the particles by means of HRTEM (Fig. 3a), allows observing lattice fringes going all along the particle long axis and regularly spaced at 3.4 $\AA$ from each other (see intensity profile in the inset of Fig. 3a). This distance corresponds to the (200) interplanar distance of the tetragonal $\mathrm{LuPO}_{4}$ phase and indicates that the $a$ axis of the unit cell is parallel to the short axis of the particle. In order to learn about the orientation of the other two axis of the unit cell, we have calculated the digital diffraction pattern (DDP) from the area selected in Fig. 3b and magnified in Fig. 3c. The DDP (shown as inset in Fig. 3c) shows a set of spots that correspond to the [010] zone axis of tetragonal $\mathrm{LuPO}_{4}$. The spots assigned to the (200) planes of such phase are aligned perpendicular to the long axis of the particles while the ones assigned to the (101) planes are forming $48^{\circ}$ with the former. These data confirm that the $a$-axis of the unit cell is parallel to the short axis of the particle and indicate that the $c$-axis is parallel to the long axis of the particle.

\subsection{Influence of the synthesis conditions on the morphology of the precipitated particles}

The set of experimental conditions used above for the synthesis of $\mathrm{LuPO}_{4}$ nanoparticles (lutetium acetate and $\mathrm{H}_{3} \mathrm{PO}_{4}$ as $\mathrm{Lu}^{3+}$ and phosphate source, respectively, at $0.025 \mathrm{M}$ 


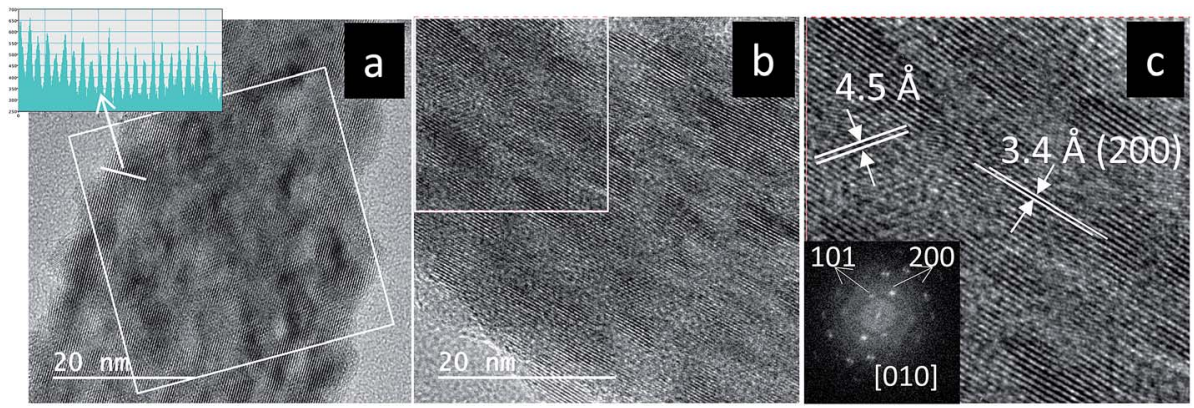

Fig. 3 (a) HRTEM image of a single $\mathrm{LuPO}_{4}$ nanoparticle and intensity profile along the white line showing a regular separation between peaks of 3.5 $\AA$. ( $b$ and c) Magnification of the quadrangles shown in ( $a$ and $b)$, respectively where two families of planes can be observed. The inset is the digital diffraction pattern obtained from the HRTEM image shown in (c).

and $0.1 \mathrm{M}$ concentrations, respectively, BG as polyol, and $180{ }^{\circ} \mathrm{C}$ aging temperature) is essential to obtain uniform nanoparticles. The variation of either the $\mathrm{Lu}^{3+}$ or phosphate source or their concentrations, or the polyol nature led either to bigger elongated particles, to irregular particles, or to highly aggregated nanoparticles, as observe in Fig. S1† and detailed below.

As observed in Fig. S1a, $\dagger$ an important change in morphology (from uniform, dispersed ovoid particles to aggregated nanorods) was observed when lutetium acetate was replaced by lutetium acetylacetonate, which indicates a strong influence of the $\mathrm{Lu}^{3+}$ precursor on the morphological characteristics of the product, in agreement with the literature on particle synthesis in polyol medium. ${ }^{20}$ On the other hand, the decrease of the $\mathrm{Lu}^{3+}$ concentration from $0.025 \mathrm{M}$ to $0.010 \mathrm{M}$ resulted in an increase of particle size $(\sim 350 \mathrm{~nm} \times$ $\sim 200 \mathrm{~nm}$, Fig. $\mathrm{S} 1 \mathrm{~b} \dagger)$. This result can be understood in terms of the classical theory of solution nucleation and particle growth. ${ }^{21}$ As the $\mathrm{Lu}^{3+}$ concentration decreases, the precipitation reaction is expected to become slower thus decreasing the number of nuclei which further grow to a higher size. Fig. S2 $\dagger$ is a plot of the particle length $v s$. $\mathrm{Lu}^{3+}$ concentration showing an exponential decay of the size as the $\mathrm{Lu}^{3+}$ concentration increases, as expected from the afore mentioned theory of nucleation and growth. The effect of the phosphate source was analyzed using $\mathrm{NaH}_{2} \mathrm{PO}_{4}$ and $\mathrm{NH}_{4} \mathrm{H}_{2} \mathrm{PO}_{4}$ as alternative $\left[\mathrm{PO}_{4}\right]^{-3}$ precursors. However, both compounds were insoluble in $\mathrm{BG}$ in the concentration required for comparison with $\mathrm{H}_{3} \mathrm{PO}_{4}(0.1$ $\mathrm{M})$. We used alternatively the ionic liquid ([BMIM] $\left.\mathrm{PO}_{4}\right)$. Fig. S1c $\uparrow$ shows the product obtained after changing the phosphate source to the ionic liquid and keeping the rest of experimental conditions unchanged. It consists, essentially, of tiny aggregated particles whose formation could be due to the possible adsorption of the BMIM cations on the $\mathrm{LuPO}_{4}$ nuclei, thus limiting their growth. The concentration of $\mathrm{H}_{3} \mathrm{PO}_{4}$ had also a strong influence on the particle size. The decrease of the concentration to $0.05 \mathrm{M}$ resulted in the formation of highly aggregated nanoparticles (Fig. S1d $\dagger$ ). The significant change in particle morphology and size with decreasing $\mathrm{H}_{3} \mathrm{PO}_{4}$ concentration indicates a decrease of the precipitation rate, probably as a consequence of the decrease in the amount of phosphate anions available for precipitation. A similar effect was observed in the synthesis of $\mathrm{GdPO}_{4}$ particles. ${ }^{\mathbf{1 2}}$

Finally, the nature of the solvent was also found to be a critical factor for the formation of uniform particles since the use of glycerol (Gly) or ethylene glycol (EG) instead of BG, led to strongly aggregated, ill-defined nanoparticles (Fig. S1e and $\mathrm{S} 1 \mathrm{f}^{\dagger}$ ). These morphological differences could be assigned to the different values of viscosity and dielectric constant of the solvents, which may affect the diffusion processes required for nucleation and particle growth.

In summary, it can be concluded that the use of a solution of lutetium acetate and $\mathrm{H}_{3} \mathrm{PO}_{4}$ in $\mathrm{BG}$ allows obtaining ovoid particles whose size can be tuned by changing the $\mathrm{Lu}^{3+}$ or the $\left(\mathrm{PO}_{4}\right)^{3-}$ concentration in the precursor solution.

\subsection{Formation mechanism of the $\mathrm{LuPO}_{4}$ nanoparticles}

To elucidate the mechanism for the formation of the $\mathrm{LuPO}_{4}$ nanoparticles shown in Fig. 1, time-dependent experiments ( 4 minutes, 10 minutes and 30 minutes) were carried out in the appropriate synthesis conditions (lutetium acetate and $\mathrm{H}_{3} \mathrm{PO}_{4}$ solution in BG, $0.025 \mathrm{M}$ and $0.1 \mathrm{M}$, respectively, aged at $180^{\circ} \mathrm{C}$ ). TEM images of the corresponding intermediates are shown in Fig. 4 along with the XRD pattern of each reaction product. After 4 minutes the TEM images showed an ill-defined precipitate with a certain degree of long range organization, as revealed by the three broad features observed in the corresponding XRD pattern. Precipitation of small ovoid particles was only observed after 10 minutes aging, which coexisted with the ill-defined precipitate. These new particles gave rise to sharp reflections in the XRD pattern, corresponding to tetragonal $\mathrm{LuPO}_{4}$, which stand above the broad features characteristic of the first precipitate. Finally, after aging the solution for 30 minutes, the reaction product consisted, exclusively, of $\mathrm{LuPO}_{4}$ nanoparticles, while the corresponding XRD pattern only showed the reflections characteristic of tetragonal $\mathrm{LuPO}_{4}$.

The formation of a precipitate in the first stages of the reaction seemed to indicate that the formation of the final particles would proceed through an aggregation process. However, the HRTEM and DDP study presented above suggests 

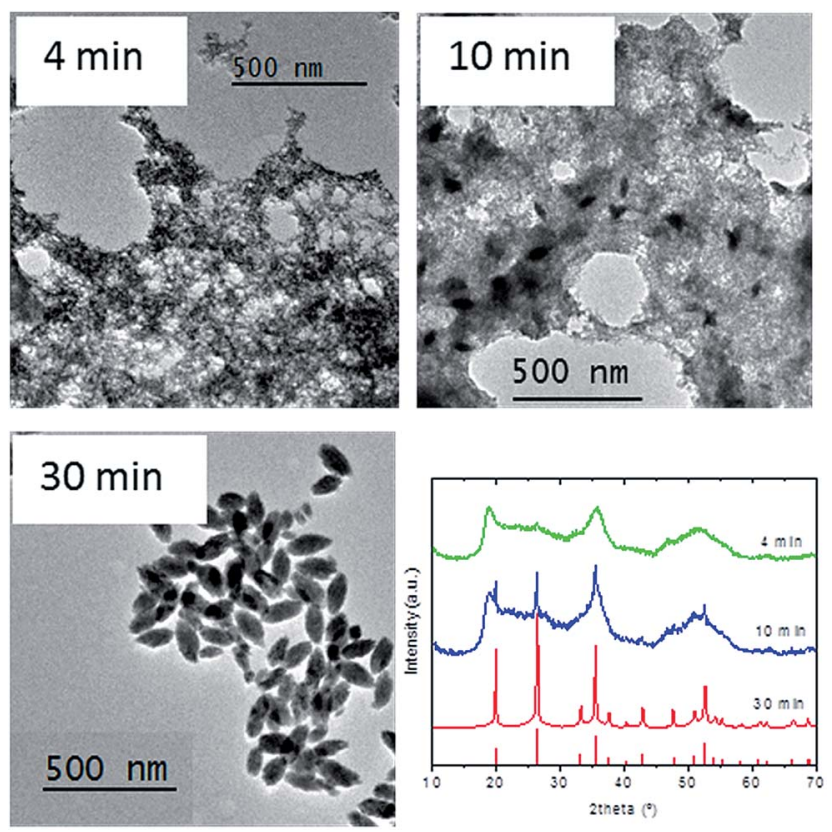

Fig. 4 TEM micrographs of three different intermediates in the formation of the $\mathrm{LuPO}_{4}$ nanoparticles. Also shown are the XRD patterns corresponding to such intermediates and PDF 00-043-0003 of tetragonal $\mathrm{LuPO}_{4}$

that the mechanism for the formation of the nanoparticles implied dissolution of the gel-like precipitate and recrystallization to form the $\mathrm{LuPO}_{4}$ nanoparticles. A similar behavior was observed for the formation of $\mathrm{Fe}_{2} \mathrm{O}_{3}$ particles. ${ }^{22}$

\subsection{Synthesis and characterization of $\mathrm{Eu}^{3+}$-doped $\mathrm{LuPO}_{4}$ nanoparticles}

We have used $\mathrm{Eu}^{3+}$ doping, as a proof on concept, to analyze the luminescent properties of the resultant $\mathrm{LuPO}_{4}$ phosphors. Four different compositions $\left(2.5 \%, 5 \%, 10 \%\right.$ and $15 \% \mathrm{Eu}^{3+}$ in $\left.\mathrm{LuPO}_{4}\right)$ were synthesized using the method reported above for the undoped material, and adding the corresponding amount of europium acetate in each case. It was found that the shape of the undoped particles was reproduced for the doped samples for doping levels up to $10 \%$ (Fig. 5), although a significant decrease in particle size was observed with increasing the $\mathrm{Eu}^{3+}$ content (Table 1). At $\mathrm{Eu}^{3+}$ contents of $15 \%$, the particles lost their original form and exhibited a high degree of aggregation (Fig. S3†). The latter composition was, therefore, not included in the luminescence study.

To confirm the presence of $\mathrm{Eu}^{3+}$ in the $\mathrm{LuPO}_{4}$ crystal structure, the XRD patterns of the $\mathrm{LuPO}_{4}$ particles doped with different $\mathrm{Eu}^{3+}$ contents (Fig. S4†) were analyzed by the Rietveld method to obtain the corresponding unit cell volumes. The values obtained have been plotted in Fig. 6, where a linear increase of the volume can be observed with increasing $\mathrm{Eu}^{3+}$ content, in agreement with the bigger ionic radius of $\mathrm{Eu}^{3+}$ compared with that of $\mathrm{Lu}^{3+} \cdot{ }^{23}$ This behavior satisfies Vegard's law ${ }^{24}$ and indicates that $\mathrm{Eu}^{3+}$ is replacing $\mathrm{Lu}^{3+}$ in the crystallographic sites of the $\mathrm{LuPO}_{4}$ structure.
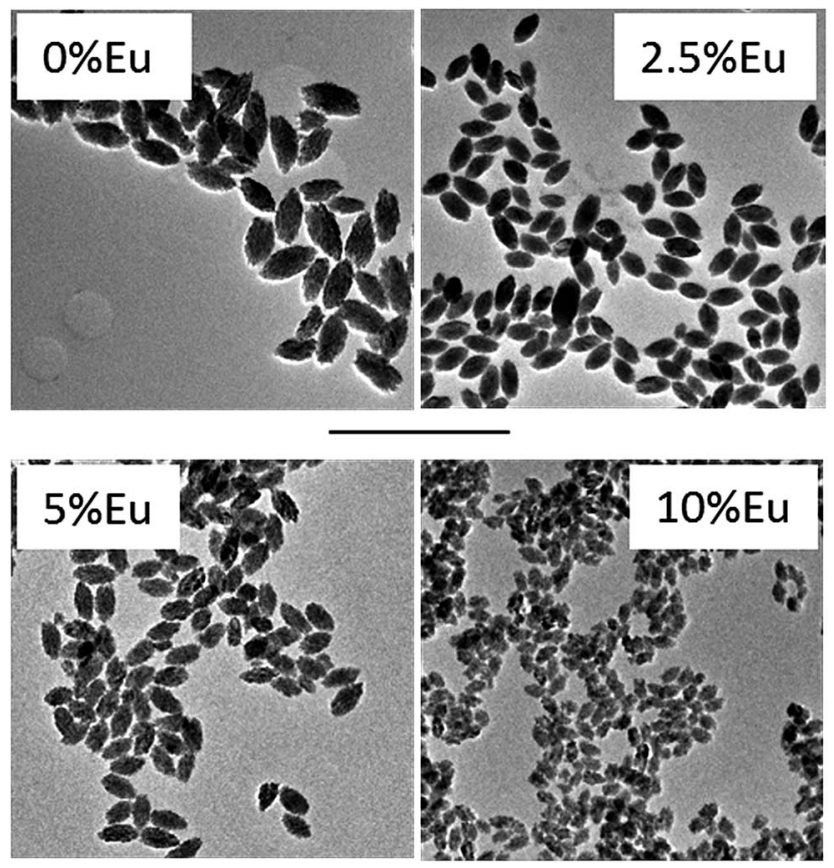

Fig. 5 TEM micrographs of the $\mathrm{Eu}^{3+}$-doped $\mathrm{LuPO}_{4}$ nanoparticles with different $\mathrm{Eu}^{3+}$ contents. The bar size is $200 \mathrm{~nm}$.

Table 1 Dimensions of $\mathrm{LuPO}_{4}$ nanoparticles doped with different $\mathrm{Eu}^{3+}$ contents. The values have been obtained from counting around a hundred particles of each type in TEM images

\begin{tabular}{lll}
\hline Eu content/\% & Length $(\mathrm{sd})(\mathrm{nm})$ & Width$(\mathrm{sd})(\mathrm{nm})$ \\
\hline 0 & $130(19)$ & $62(9)$ \\
2.5 & $90(8)$ & $45(5)$ \\
5 & $85(8)$ & $40(5)$ \\
10 & $50(5)$ & $30(3)$
\end{tabular}

\subsection{Study of the luminescence properties of the $\mathrm{Eu}^{3+}$-doped $\mathrm{LuPO}_{4}$ nanoparticles}

The excitation spectra of the $\mathrm{Eu}^{3+}$-doped $\mathrm{LuPO}_{4}$ nanoparticles were recorded by monitoring the emission of the characteristic ${ }^{5} \mathrm{D}_{0}{ }^{-}{ }^{7} \mathrm{~F}_{1}$ transition of $\mathrm{Eu}^{3+}$ at $593 \mathrm{~nm}$. Fig. 7 (top) shows the excitation spectrum corresponding to the $5 \% \mathrm{Eu}^{3+}$-doped sample. The rest of compositions showed very similar excitation spectra to this one. They all exhibited a set of sharp and well defined excitation bands, the most intense at $395 \mathrm{~nm}$, which correspond to the $\mathrm{f}-\mathrm{f}$ electronic transitions characteristic of the $\mathrm{Eu}^{3+}$ ions ${ }^{25}$ and that have been assigned in the figure. The high intensity band at $\lambda<270 \mathrm{~nm}$, partially observed in the spectrum, is known as Eu-O charge transfer band and it is due to the electron transfer from the fully occupied $2 \mathrm{p}^{6}$ orbital of the $\mathrm{O}^{2-}$ anion and the partially occupied $4 \mathrm{f}$ orbitals of $\mathrm{Eu}^{3+} .^{26}$

The emission spectra of the $\mathrm{Eu}^{3+}$-doped $\mathrm{LuPO}_{4}$ nanoparticles (molar\% $\mathrm{Eu}^{3+}$ from $2.5 \%$ to $10 \%$ ) recorded after excitation at $395 \mathrm{~nm}$, are shown in Fig. 7 (bottom). The observed emission bands correspond to the well-known ${ }^{5} \mathrm{D}_{0}{ }^{-} \mathrm{F}_{J}(J=0,1,2,3,4)$ transitions of $\mathrm{Eu}^{3+}$. The ${ }^{5} \mathrm{D}_{0}-{ }^{7} \mathrm{~F}_{1}$ transition is due to the magnetic dipole (MD) transition of $\mathrm{Eu}^{3+}$ ions and it is 


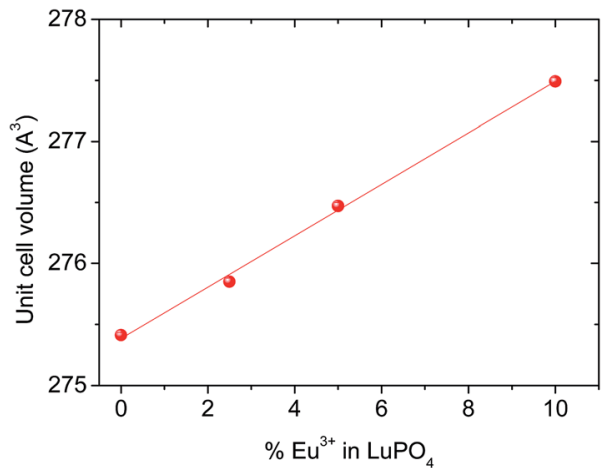

Fig. 6 Unit cell volume of the $\mathrm{Eu}^{3+}$-doped $\mathrm{LuPO}_{4}$ nanoparticles as a function of $\mathrm{Eu}^{3+}$ concentration calculated by the Rietveld analysis of their corresponding XRD patterns. The error bars are of the symbol size.
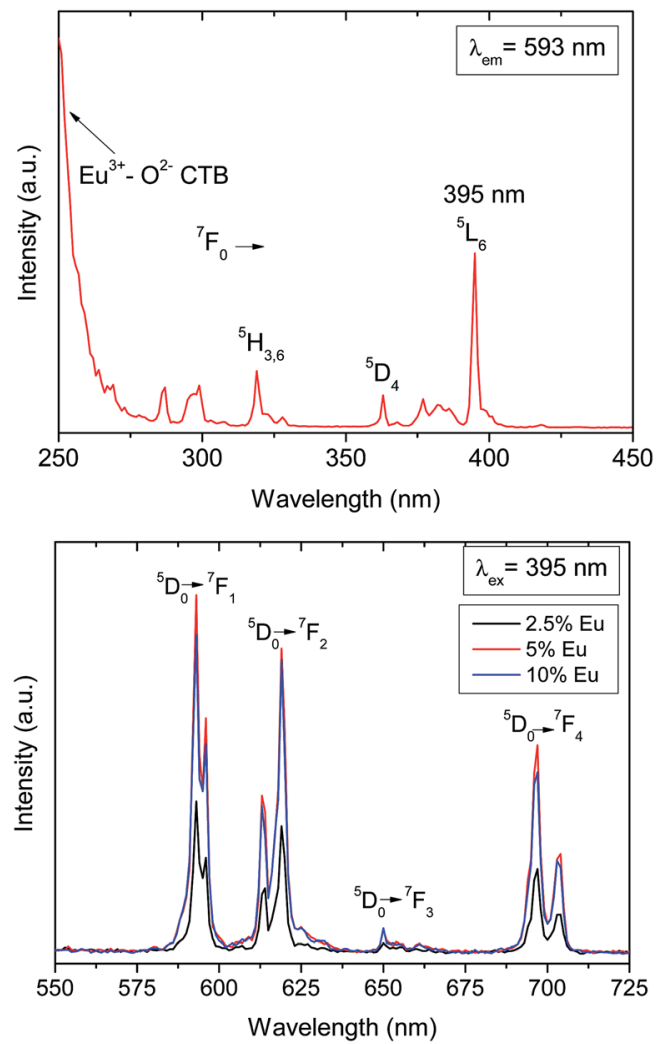

Fig. 7 (Top) Excitation spectrum of the 5\% Eu ${ }^{3+}$-doped $\mathrm{LuPO}_{4}$ nanoparticles recorded at an emission wavelength of $593 \mathrm{~nm}$. (Bottom) Emission spectra of different $\mathrm{Eu}^{3+}$-containing $\mathrm{LuPO}_{4}$ nanoparticles recorded at an excitation wavelength of $395 \mathrm{~nm}$.

independent of the symmetry of the $\mathrm{Eu}^{3+}$ site. However, the ${ }^{5} \mathrm{D}_{0}-{ }^{7} \mathrm{~F}_{2}$ transition is a forced electric dipole (ED) transition, hypersensitive to the site symmetry of the $\mathrm{Eu}^{3+}$ ions. ${ }^{1}$ The intensity of both emission bands is very similar (ratio of the integral areas $\mathrm{MD} / \mathrm{ED}=1.04$ ), as observed also in Eu-doped $\mathrm{LaPO}_{4}$ particles. ${ }^{19}$ This result indicates that the $\mathrm{Eu}^{3+}$ ions are located at a site without inversion center, ${ }^{27}$ in good agreement with the tetragonal $I_{1} /$ amd structure of $\mathrm{LuPO}_{4}$, which locates the Lu atom at a 4 a site with $D_{2 \mathrm{~d}}$ point group symmetry. ${ }^{18}$

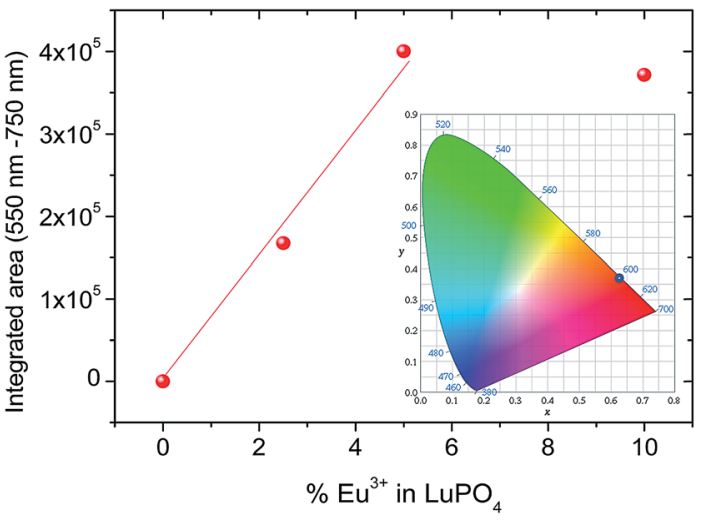

Fig. 8 Integrated area of the emission spectra of the $\mathrm{Eu}^{3+}$-doped $\mathrm{LuPO}_{4}$ nanoparticles as a function of $\mathrm{Eu}^{3+}$ concentration. The inset is a $\mathrm{CIE}$ diagram showing the chromatic coordinates of the $\mathrm{Eu}^{3+}$-doped particles.

Although the emission spectra of all compositions were very similar to each other from a qualitative point of view, their absolute intensities varied significantly with increasing $\mathrm{Eu}^{3+}$ content. Fig. 8 shows the integrated intensity of the emission spectrum obtained for each composition as a function of the $\mathrm{Eu}^{3+}$ content. The emission intensity increased linearly with increasing $\mathrm{Eu}^{3+}$ content, reaching a maximum at the $\mathrm{LuPO}_{4}: 5 \%$ $\mathrm{Eu}^{3+}$ composition. The observed increase in luminescence intensity is clearly due to the progressive increase of emission centers as the doping concentration increases. After reaching a maximum, the luminescence intensity decreases with increasing the $\mathrm{Eu}^{3+}$ doping level. This fact, known as concentration quenching effect, is due to the energy transfer between adjacent luminescent centers and becomes significant when the $\mathrm{Eu}-\mathrm{Eu}$ distance decreases as a result of the increase in doping level. ${ }^{1}$

The colloidal suspensions of the $\mathrm{Eu}^{3+}$-doped $\mathrm{LuPO}_{4}$ nanoparticles dispersed in water emit a strong red-orange luminescence under UV radiation with CIE coordinates $(x=0.63, x=$ 0.37) at any $\mathrm{Eu}^{3+}$ content (Fig. 8, inset).

\subsection{Functionalization and colloidal stability of $\mathrm{Eu}^{3+}$-doped $\mathrm{LuPO}_{4}$ nanoparticles}

The optimum $\mathrm{Eu}^{3+}$-doping composition found in the luminescence analysis was further used to test the functionalization capacity of the particles. Polyacrylic acid (PAA) was used as the functionalizing molecule (see the followed procedure in the Experimental section) due to its highly hydrophilic nature, which is appropriate for applications in the biotechnology field. ${ }^{28}$ The success of the functionalization process was proved by FTIR spectroscopy. The FTIR spectra of the particles, before and after the functionalization process, are shown in Fig. 9 (top), together with the FTIR spectrum of pure PAA. The spectrum of the nude particles consisted of a group of bands at $<1200 \mathrm{~cm}^{-1}$ corresponding to the stretching and bending vibrations of the phosphate group. The bands at $3420 \mathrm{~cm}^{-1}$ and $1635 \mathrm{~cm}^{-1}$ are due to adsorbed water on the particles surface. ${ }^{14}$ The FTIR spectrum of the PAA-treated particles exhibited, in addition to the bands found in the spectrum of the nude 

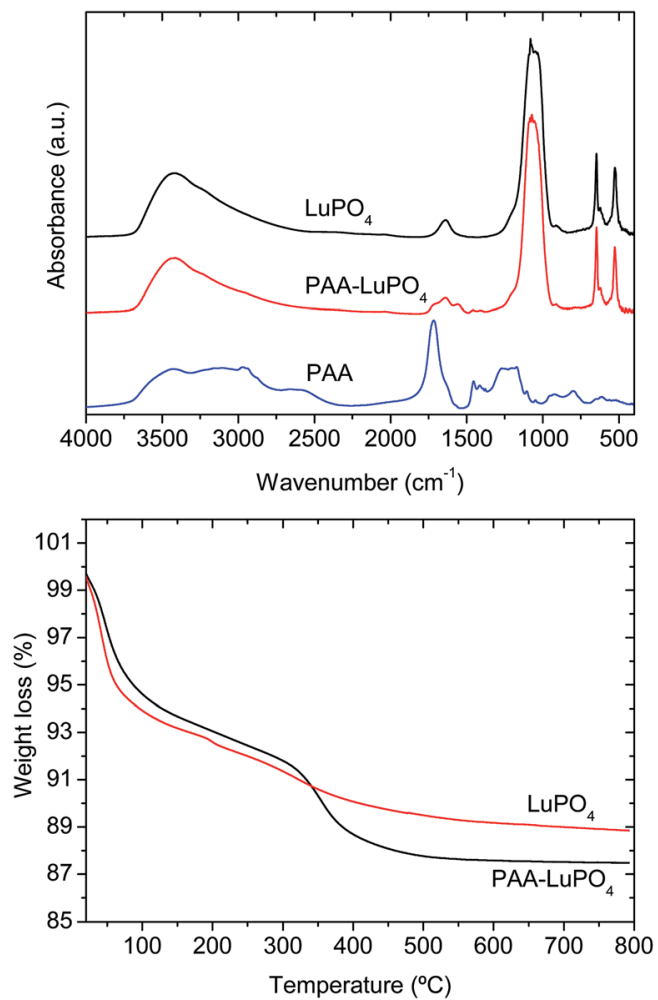

Fig. 9 (Top) FT-IR spectra of the $5 \% \mathrm{Eu}^{3+}$-doped $\mathrm{LuPO}_{4}$ particles before and after the functionalization treatment with PAA, and FT-IR spectrum of pure PAA. (Bottom) Thermogravimetry curves of the $5 \%$ $\mathrm{Eu}^{3+}$-doped $\mathrm{LuPO}_{4}$ particles before and after the functionalization treatment with PAA.

particles, some extra signals in the $1800-1350 \mathrm{~cm}^{-1}$ region that correspond to the stretching vibrations of the carboxylate anion in the PAA polymer. This finding confirmed the incorporation of the polymer onto the surface of the nanoparticles, as concluded for other lanthanide-based particles. ${ }^{29}$ The zeta potential of the particles before and after functionalization was also a measure of the success of the process. Thus, while a value of $0.3 \mathrm{mV}$ was registered in the nude particles at $\mathrm{pH}=4$, a charge reversal (zeta potential $=-38.5 \mathrm{mV}$ ) was observed for the treated particles at the same pH. Finally, the amount of PAA species attached to the nanoparticles surface was quantified by thermogravimetric analyses (TGA). The TGA curve obtained for the PAA-functionalized $\mathrm{Eu}^{3+}: \mathrm{LuPO}_{4}$ nanoparticles is shown in Fig. 9 (bottom), along with the curve corresponding to the particles before functionalization. While the latter consists of a mass loss due to the release of water ( $\sim 11 \%$ of the total mass) in the temperature range 20 $300{ }^{\circ} \mathrm{C}$, the TGA curve of the PAA functionalized $\mathrm{Eu}^{3+}: \mathrm{LuPO}_{4}$ nanoparticles exhibited an additional weight loss (from $300^{\circ}$ up to $700{ }^{\circ} \mathrm{C}$ ) that can be associated to the decomposition of the adsorbed PAA and represents $\sim 4 \%$ of the total mass.

Finally, the colloidal stability of the PAA-functionalized $\mathrm{Eu}^{3+}: \mathrm{LuPO}_{4}$ particles was checked in two different biological media: MES (at $\mathrm{pH}=6.5$ ) and PBS (at $\mathrm{pH}=7.2$ ) (see details in the Experimental section). The average hydrodynamic diameter of the nanoparticles remained unaltered $(\sim 90 \mathrm{~nm})$ during at least 15 days of aging in both media (Fig. 10), which indicated
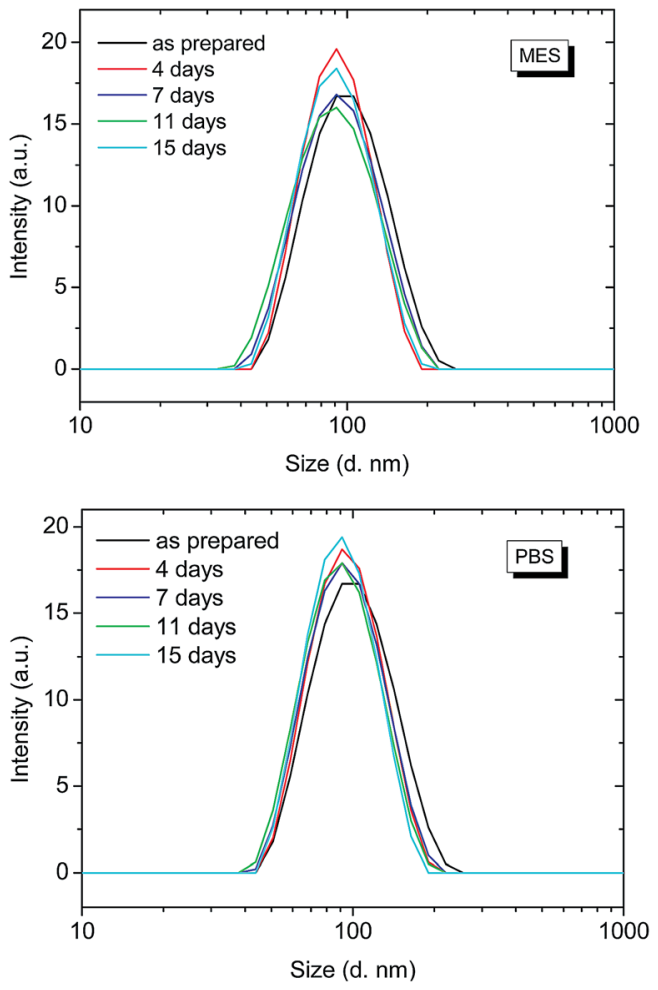

Fig. 10 Hydrodynamic diameter obtained by DLS measurements for the $5 \% \mathrm{Eu}^{3+}$-doped $\mathrm{LuPO}_{4}$ particles functionalized with PAA and dispersed in MES ( $\mathrm{pH}$ 6.5) and PBS ( $\mathrm{pH}=7.2$ ) (top and bottom, respectively), after different aging periods at room temperature.

an excellent colloidal stability of the particles and their suitability for applications in biotechnology.

\section{Concluding remarks}

Uniform, ovoid $\mathrm{Eu}^{3+}$-doped lutetium orthophosphate nanoparticles $(85 \mathrm{~nm} \times 40 \mathrm{~nm})$ have been synthesized using a quick, simple, and green method based on the homogeneous precipitation of ions in a butylene glycol solution containing, exclusively, $\mathrm{Lu}^{3+}$ and $\mathrm{Eu}^{3+}$ acetates $(0.025 \mathrm{M})$ and $\mathrm{H}_{3} \mathrm{PO}_{4}(0.1 \mathrm{M})$ aged at $180{ }^{\circ} \mathrm{C}$. The nanoparticles consisted of single crystals of tetragonal Eu:LuPO 4 and DLS measurements revealed that the particles were well dispersed in aqueous solution. The $5 \% \mathrm{Eu}-$ doped $\mathrm{LuPO}_{4}$ nanoparticles, were successfully functionalized with polyacrylic acid and showed a very high colloidal stability in two different biological buffers (MES, pH 6.5 and PBS, $\mathrm{pH}=$ 7.2). These properties make the PAA-functionalized Eu:LuPO nanoparticles good candidates as optical labels for in vitro applications. Also their uniformity and luminescence characteristics indicate that the Eu:LuPO${ }_{4}$ nanoparticles are excellent materials for laser applications.

\section{Acknowledgements}

Supported by MEC (Project MAT2011-23593), Junta de Andalucía (JA FQM 06090) and CSIC (201460E005). Olga Montes is gratefully ackowledged for help with Tecnai 20 instrument. 


\section{References}

1 G. Blasse and B. C. Grabmaier, Luminescent Materials, Springer Verlag, Berlin, 1994.

2 E. L. Cates, S. L. Chinnapongse, J. H. Kim and J. H. Kim, Environ. Sci. Technol., 2012, 45, 12316-12328.

3 M. Shang, C. Li and J. Lin, Chem. Soc. Rev., 2014, 43, 13721386.

4 D. K. Chatterjee, M. K. Gnanasammandhan and Y. Zhang, Small, 2010, 6, 2781-2795.

5 X. Li, F. Zhang and D. Zhao, Nano Today, 2013, 8, 643-676.

6 L. Zhang, L. Fu, X. Yang, Z. Fu, X. Qi and Z. Wu, J. Mater. Chem. C, 2014, 2, 9149-9158.

7 K. Hickmann, V. John, A. Oertel, K. Koempe and M. Haase, J. Phys. Chem. C, 2009, 113, 4763-4767.

8 Y. Xing, M. Li, S. A. Davis and S. Mann, J. Phys. Chem. B, 2006, 110, 1111-1113.

9 R. X. Yan, X. M. Sun, X. Wang, Q. Peng and Y. D. Li, Chem.Eur. J., 2005, 11, 2183-2195.

10 A. I. Becerro, S. Rodriguez-Liviano, A. J. Fernandez-Carrion and M. Ocaña, Cryst. Growth Des., 2013, 13, 526-535.

11 W. Ren, G. Tian, L. J. Zhou, W. Y. Yin, L. Yan, S. Jin, Y. Zu, S. J. Li, Z. J. Gu and Y. L. Zhao, Nanoscale, 2012, 4, 3754-3760.

12 S. Rodriguez-Liviano, A. I. Becerro, D. Alcantara, V. Grazú, J. M. de la Fuente and M. Ocaña, Inorg. Chem., 2013, 52, 647-654.

13 Y. Li, T. Chen, W. Tan and D. R. Talham, Langmuir, 2014, 30, 5873-5879.

14 Y. Gao, M. Fan, Q. Fang, B. Song and W. Jiang, J. Nanosci. Nanotechnol., 2013, 13, 6644-6652.
15 S. Heer, O. Lehmann, M. Haase and H. U. Güdel, Angew. Chem., Int. Ed., 2003, 42, 3179-3182.

16 O. Lehmann, H. Meyssamy, K. Kömpe, H. Schnablegger and M. Haase, J. Phys. Chem. B, 2003, 107, 7449-7453.

17 B. J. Shen, L. D. Sun, J. D. Zhu, L. H. Wei and C. H. Yan, Adv. Funct. Mater., 2010, 20, 3708-3714.

18 Y. X. Ni, J. M. Hughes and A. N. Mariano, Am. Mineral., 1995, 80, 21-26.

19 N. O. Nuñez and M. Ocaña, Nanotechnology, 2007, 18, 455606.

20 N. O. Nuñez, S. R. Liviano and M. Ocaña, J. Colloid Interface Sci., 2010, 349, 484-491.

21 V. K. Lamer and R. J. Dinegar, J. Am. Chem. Soc., 1950, 72, 4847-4854.

22 M. Ocaña, M. P. Morales and C. J. Serna, J. Colloid Interface Sci., 1995, 171, 85-91.

23 R. D. Shannon, Acta Crystallogr., Sect. A: Cryst. Phys., Diffr., Theor. Gen. Crystallogr., 1976, 32, 751-767.

24 L. Vegard, Z. Phys., 1921, 5, 17-26.

25 R. Yan and Y. Li, Adv. Funct. Mater., 2005, 15, 763-770.

26 S. Rodriguez-Liviano, F. J. Aparicio, T. C. Rojas, A. B. Hungría, L. E. Chinchilla and M. Ocaña, Cryst. Growth Des., 2012, 12, 635-645.

27 P. A. Tanner, Chem. Soc. Rev., 2013, 42, 5090-5101.

28 I. Y. Toth, E. Illes, R. A. Bauer, D. Nesztor, M. Szekeres, Y. Zupko and E. Tomba, Langmuir, 2012, 28, 16638-16646.

29 N. O. Nuñez, S. Rivera, D. Alcantara, J. M. de la Fuente, J. García-Sevillano and M. Ocaña, Dalton Trans., 2013, 42, 10725-10734. 